\title{
Composição e Similaridade da Flora Associada A Sítios antropizados do Município de João Pessoa - Paraíba ${ }^{1}$
}

\author{
Composition and Similarity of Flora Associated with Anthropogenic Sites in the City of João \\ Pessoa - Paraiba
}

MACHADO FILHO, H.O. ${ }^{2,3}$, COUTO, E.A. ${ }^{3}$, BEZERRA, C.P. ${ }^{3,4}$ e MELO, J.I.M. ${ }^{5}$

\begin{abstract}
RESUMO - Neste estudo é apresentada a composição e similaridade florística de espécies vasculares sinantrópicas em uma área periurbana antropizada, no município de João Pessoa, Estado da Paraíba, e sua relação com outras floras sinantrópicas. Foram encontradas 98 espécies, em 75 gêneros e 30 famílias. Entre as espécies, 41,84\% são cosmopolitas; 29,6\%, pantropicais; 13,3\% ocorrem apenas no cone sul da América; 8,2\%, neotropicais; 5,1\%, gondwânicas; e $2 \%$ ocorrem em toda a América. Asteraceae e Poaceae foram as famílias mais representativas em número de espécies. Os resultados foram comparados com os de outros levantamentos florísticos correlatos, utilizando o índice de similaridade de Sörensen, e observou-se que não é somente a proximidade geográfica entre os locais estudados que reflete uma maior similaridade florística entre as áreas, e sim a amplitude dos táxons e a presença de micro-habitat semelhante. A área de estudo está relacionada a uma área inserida na região Centro-Oeste do Brasil.
\end{abstract}

Palavras-chave: florística, plantas sinantrópicas, Nordeste, Brasil.

\begin{abstract}
This study presents the floristic composition and similarity of synanthropic vascular plant species in an anthropogenic peri-urban area in the city of João Pessoa, in the state of Paraiba, and how it is related to other synanthropic floras. A total of 98 species in 75 genera and 30 families were found. Among the species, 41.84\% are cosmopolitan, 29.6\% pantropical, 13.3\% occur only in the Southern Cone of America, 8.2\% are Neotropical, 5.1\% gondwanic and 2\% occurs across America. Asteraceae and Poaceae were the families with the biggest number of species. The results were compared with other related floristic surveys using Sörensen's similarity coefficient, and it was observed that not it is only the geographical proximity of the two locations that reflects a higher floristic similarity between areas but also the extent of the taxa and the presence of a similar microhabitat. The study focuses on an area located in the Brazil's Midwest region.
\end{abstract}

Keywords: floristics, synanthropic plants, northeast, Brazil.

\section{INTRODUÇÃO}

A vegetação associada aos ambientes tipicamente antropizados ou sinantrópicos tem como características específicas a facilidade de sobrevivência e dispersão nos ambientes perturbados pela ação degradadora humana (Pedrotti \& Guarim-Neto, 1998). Essas comunidades, também chamadas de ruderais (ambientes urbanos) ou "daninhas" (ambientes rurais), se desenvolvem sob condições adversas àquelas que compõem ecossistemas naturais, incluindo diferenças nas próprias populações e nos ambientes físicos que elas

Recebido para publicação em 22.2.2014 e aprovado em 9.8.2014.

2 Universidade Federal Rural de Pernambuco, Recife-PE, Brasil, < hermes@ifpb.edu.br>; ${ }^{3}$ Instituto Federal de Educação Ciência e Tecnologia, João Pessoa-PB, Brasil; ${ }^{4}$ Universidade Federal do Rio Grande do Norte, Natal, RN, Brasil; ${ }^{5}$ Universidade Estadual da Paraíba, Campina Grande, Paraíba-PB, Brasil. 
ocupam (Jarolímek \& Zaliberová, 1995), sendo a variável de maior importância a diferença entre as intensidades da interferência humana (Hill et al., 2002).

Esses organismos crescem subespontaneamente em áreas ligadas aos habitats perturbados e/ou construídos, como terrenos devastados, degradados/abandonados, caminhos, estradas, nas margens de ruas, jardins e similares, além de loteamentos urbanos, canteiros de obras urbanas, sobre muros, telhados, calçadas e áreas agrícolas, comportando-se, quase sempre, como indesejáveis.

Segundo Mennan \& Isik (2003) e Rajput et al. (2008), essas plantas, quando crescem juntamente com outras culturas, podem interferir potencialmente no seu desenvolvimento, reduzindo-lhes a produção, competindo pela extração dos elementos vitais, como água, luz, $\mathrm{CO}_{2}$ e nutrientes, e até exercendo inibição química, como, por exemplo, a alelopatia, sobre o desenvolvimento dos cultivares.

Essas comunidades de plantas podem ainda comprometer, indiretamente, culturas agrícolas, por hospedarem pragas e doenças antes de infestarem as próprias lavouras; na pecuária, as plantas daninhas interferem com as forrageiras, reduzindo a capacidade de lotação das pastagens; algumas são causadoras de ferimentos mecânicos nos animais e outras conferem gosto desagradável ao leite, se ingeridas por vacas em lactação (Pedrotti \& Guarim-Neto, 1998).

De acordo com Pitelli \& Pavani (2005), a habilidade dessas plantas sinantrópicas, quanto à sobrevivência, é atribuída principalmente a caracteres ou mecanismos de estratégias de vida, como grande agressividade competitiva, grande produção de sementes (pequenas, leves, aladas ou com ganchos), longevidade dessas sementes, entre outros aspectos. Trata-se de um grupo considerado infestante e forte competidor por espaço, sendo tratado como um tema relevante nas questões biogeográficas na atualidade (Almeida \& Freitas, 2000; Colautti \& MacIsaac, 2004).

O conhecimento sobre a flora sinantrópica é fundamental para a implantação de políticas de controle de espécies. Entretanto, os levantamentos enfocando esse grupo de plantas são ainda escassos, especialmente na região Nordeste do Brasil.

Entre os trabalhos recentes, enfocando espécies sinantrópicas, podem ser mencionados os realizados por Almeida \& Freitas (2000), Pišek et al. (2004), Schneider (2007), Shan-Huah et al. (2009), Silva et al. (2010) e Hassemer \& Trevisan (2012).

Diante do exposto, este trabalho objetivou apresentar o levantamento das espécies vegetais sinantrópicas no município de João Pessoa, Paraíba, e verificar a similaridade dessa flora com relação a outras floras de ambientes degradados, esperando-se que, pelo fato de as espécies apresentarem ampla distribuição geográfica, as floras tendam a se apresentar mais similares entre si, independentemente da distância geográfica.

\section{MATERIAL E MÉTODOS}

Área de Estudo - A Zona da Mata paraibana encontra-se no litoral do Nordeste do Brasil, no domínio da Mata Atlântica, e apresenta elevações que variam entre $37 \mathrm{e}$ $74 \mathrm{~m}$, ocupando uma área de aproximadamente $210,6 \mathrm{~km}^{2}$ (Albertino et al., 2004). O clima é quente e úmido, do tipo intertropical; a temperatura do ar é, predominantemente, elevada, sendo isotérmica de $26,6^{\circ} \mathrm{C}$, sofrendo oscilações entre $22{ }^{\circ} \mathrm{C}$ e $30{ }^{\circ} \mathrm{C}$. A pluviosidade média está em torno de 1.325,4 $\mathrm{mm}^{\mathrm{ano}}{ }^{-1}$ (AESA, 2013).

Nessas condições médias encontra-se o município de João Pessoa, que apresenta em seu território uma grande variação em termos de urbanização territorial, como bairros com características ruralistas em sua zona periférica. Os locais de coletas consistiram de bairros que apresentassem essas características periurbanas, com infraestrutura mais próxima de zonas rurais, como os bairros do Cinturão Verde - projeto governamental que desenvolve práticas agrícolas no perímetro da zona suburbana da cidade.

Floristica - Os trabalhos de campo foram feitos entre março de 2011 e outubro de 2012, a partir de caminhadas aleatórias. Os procedimentos de coleta e herborização foram baseados em Bridson \& Forman (2004), sendo obtidas amostras em estádio reprodutivo (flor 
e/ou fruto) de todos os representantes da flora vascular.

Paralelamente, foram anotados em caderneta de campo dados como: local, data, coletor e número, tipo de ambiente (ruas, calçadas, muros, pastagens, culturas), tipo de substrato, bem como informações sobre a planta, como: hábito (erva, subarbusto, arbusto, árvore, trepadeira, epífita) e coloração das estruturas reprodutivas, entre outros aspectos considerados importantes para a identificação taxonômica.

A identificação das espécies foi fundamentada, principalmente, em literatura especializada: flórulas, floras regionais, revisões taxonômicas e artigos. Entre as obras consultadas estão os trabalhos de Barroso et al. (1978, 1984, 1986), Aranha et al. (1988), Kissmann \& Groth (1997, 1999, 2000), Gonçalves \& Lorenzi (2012), Lorenzi (2008), além da Lista de Espécies da Flora do Brasil (Forzza et al., 2013).

A lista florística foi elaborada segundo o APG III (2009). Os nomes das espécies e respectivos autores foram verificados na base The Plant List (2013). Para determinar a área onde cada espécie encontrada é nativa, foram consultados, além da literatura especializada, os seguintes sites: Global Biodiversity Information Facility (2013), Global Invase Species Data Base (2013), JSTOR (2013) Plant Science, a base de plantas tropicais do Missouri Botanical Garden (MOBOT, 2013) e a Lista de Espécies da Flora do Brasil on line (Forzza et al., 2013).

Similaridade - Foram selecionados 14 trabalhos de florística de plantas ruderais/ daninhas para essa análise, incluindo ambientes tipicamente antropizados urbanos ou ambientes cultivados, com metodologia de amostragem similar à realizada no presente estudo, sendo eles: presente estudo [A], Mautone et al. (1990) [B], Gavilanes \& D'Angieri-Filho (1991) [C], Pedrotti \& GuarimNeto (1998) [D], Albertino et al. (2004) [E], Schneider \& Irgang (2005) [F], Carneiro \& Irgang (2005) [G], Souza \& Poletto (2007) [H], Silva et al. (2008) [I], Cattani (2009) [J], Silva et al. (2006) [K], Souza et al. (2003) [L], Maciel et al. (2008) [M] e Marques et al. (2010) [N].
Com base em cada lista floristica dos trabalhos selecionados, confeccionou-se uma tabela composta por dados binários (presença/ ausência), para cada espécie da Check List. Com essas informações, gerou-se uma matriz de similaridade florística. Foi utilizada a nomenclatura atual de cada táxon, a fim de evitar repetições e sinonimias.

As áreas comparadas apresentaram-se variáveis em tamanho e provavelmente tiveram esforços de coleta diferentes, porém foram incluídas listas que adotaram metodologias similares, a título de comparação florística.

Em seguida, foi realizada a análise de agrupamento e obteve-se um dendrograma. O método utilizado foi o Unweighted Pair-Group Method using Arithmetic Averages (UPGMA), que verifica a distância entre dois grupos a partir da média entre todos os pares de itens pertencentes aos grupos, sendo mais eficiente nesses casos de análise de agrupamento naturais e distintos, encontrando os $K$ vizinhos mais próximos do próximo a ser classificado e agrupando os dados (Gotelli \& Ellison, 2011).

A matriz binária de presença/ausência foi tratada no software PRIMER versão 6.0, sendo adotado o coeficiente de similaridade obtido através do índice de Sörensen (Kent \& Coker, 1995).

\section{RESULTADOS E DISCUSSÃO}

Foram registradas 98 espécies, distribuídas em 75 gêneros e 30 famílias (Tabela 1), sendo as mais representativas: Asteraceae (16 espécies) e Poaceae (14 espécies). Euphorbiaceae está representada por sete espécies; Fabaceae e Convolvulaceae, por seis; Lamiaceae e Verbenaceae, por cinco; Amaranthaceae, Cyperaceae, Rubiaceae e Solanaceae, por quatro; e Capparaceae, Piperaceae, Malvaceae e Talinaceae, por duas espécies cada. As demais famílias apresentaram uma espécie cada (Figura 1).

A predominância de espécies de Asteraceae $(16,3 \%)$ e Poaceae $(14,3 \%)$ se dá, principalmente, pelo fato de essas famílias possuírem estratégias favoráveis para se estabelecerem em diferentes ecossistemas, como: altas taxas de produção de sementes (Souza \& Valio, 2001) e mecanismos de 
Tabela 1 - Lista florística das espécies sinantrópicas registradas no munícipio de João Pessoa, Paraíba. Abreviações utilizadas para os tipos de hábitos: Erva (Erv), Subarbusto (Sub), Trepadeira (Trep), Árvore (Arv) e Arbusto (Arb). Abreviações adotadas para a distribuição geográfica: Cosmopolita (Cosmop), Americano (Amer), Gondwânica (Gondw), Pantropical (Pantrop), Neotropical (Neotr) e Endêmica (Endem)

\begin{tabular}{|c|c|c|c|}
\hline Família & Espécie & Hábito & $\begin{array}{c}\text { Distribuição } \\
\text { geográfica }\end{array}$ \\
\hline \multicolumn{4}{|c|}{ MONOCOTILEDÔNEAS } \\
\hline Commelinaceae & Commelina benghalensis L. & Erv & Cosmop \\
\hline \multirow{4}{*}{ Cyperaceae } & Cyperus esculentus L. & Erv & Cosmop \\
\hline & Cyperus surinamensis Rottb. & Erv & Amer \\
\hline & Kyllinga odorata Vahl & Erv & Cosmop \\
\hline & Rhynchospora corymbosa (L.) Britton & Erv & Cosmop \\
\hline \multirow{14}{*}{ Poaceae } & Cenchrus echinatus L. & Erv & Cosmop \\
\hline & Chloris barbata Sw. & Erv & Cosmop \\
\hline & Cynodon dactylon (L.) Pers. & Erv & Cosmop \\
\hline & Dactyloctenium aegyptium ( L.) Willd. & Erv & Cosmop \\
\hline & Digitaria ciliaris (Retz.) Koeler & Erv & Cosmop \\
\hline & Digitaria horizontalis Willd. & Erv & Gondw \\
\hline & Digitaria insularis (L.) Fedde & Erv & Pantrop \\
\hline & Eleusine indica (L.) Gaertn. & Erv & Cosmop \\
\hline & Eragrostis airoides Nees & Erv & Neotr \\
\hline & Eragrostis ciliaris (L.) R. BR. & Erv & Pantrop \\
\hline & Melinis repens (Willd.) Zizka & Erv & Cosmop \\
\hline & Panicum pernambucense (Spreng.) Mez ex Pilg. & Erv & Neotr \\
\hline & Paspalum modestum $\mathrm{Mez}$ & Erv & Endem \\
\hline & Pennisetum purpureum Schumach. & Erv & Cosmop \\
\hline \multicolumn{4}{|c|}{ (EU-) DICOTILEDÔNEAS } \\
\hline \multirow{4}{*}{ Amaranthaceae } & Alternanthera ficoidea (L.) P. Beauv. & Erv & Pantrop \\
\hline & Alternanthera micrantha R.E. Fr. & Erv & Pantrop \\
\hline & Amaranthus deflexus L. & Sub & Cosmop \\
\hline & Amaranthus spinosus L. & Sub & Cosmop \\
\hline Aristolochiaceae & Aristolochia arcuata Mast. & Trep & Endem \\
\hline \multirow{16}{*}{ Asteraceae } & Ageratum conyzoides L. & Erv & Cosmop \\
\hline & Bidens pilosa $\mathrm{L}$. & Erv & Cosmop \\
\hline & Bidens subalternans DC. & Erv & Cosmop \\
\hline & Bidens sulphurea (Cav. Sch.) Bip. & Erv & Gondw \\
\hline & Centratherum punctatum Cass. & Erv & Cosmop \\
\hline & Conocliniopsis prasiifolia (DC.) R.M. King \& H.Rob. & Erv & Endem \\
\hline & Conyza bonariensis (L.) Cronquist & Sub & Cosmop \\
\hline & Eclipta prostrata (L.) L. & Sub & Cosmop \\
\hline & Emilia fosbergii Nicolson & Erv & Pantrop \\
\hline & Emilia sonchifolia (L.) DC. Ex Wight. & Erv & Cosmop \\
\hline & Galinsoga parviflora Cav. & Erv & Cosmop \\
\hline & Mikania cordifolia (L.f.) Willd. & Trep & Neotr \\
\hline & Porophyllum ruderale (Jacq.) Cass. & Erv & Neotr \\
\hline & Praxelis diffusa (Rich.) Pruski & Erv & Endem \\
\hline & Sphagneticola trilobata (L.) Pruski & Trep & Cosmop \\
\hline & Tridax procumbens (L.) L. & Erv & Cosmop \\
\hline Boraginaceae & Heliotropium indicum L. & Erv & Cosmop \\
\hline \multirow{2}{*}{ Capparaceae } & Cleome affinis DC. & Erv & Endem \\
\hline & Cleome spinosa Jacq. & Sub & Cosmop \\
\hline \multirow{6}{*}{ Convolvulaceae } & Ipomoea asarifolia (Desr.) Roem. \& Schult. & Trep & Gondw \\
\hline & Ipomoea fimbriosepala Choisy & Trep & Pantrop \\
\hline & Ipomoea hederifolia $\mathrm{L}$. & Trep & Cosmop \\
\hline & Ipomoea indivisa (Vell.) Hallier f. & Trep & Endem \\
\hline & Ipomoea pes-caprae (L.) R. Br. & Erv & Cosmop \\
\hline & Merremia umbellata L. Hallier f. & Trep & Pantrop \\
\hline
\end{tabular}


Tabela 1, cont.

\begin{tabular}{|c|c|c|c|}
\hline Família & Espécie & Hábito & $\begin{array}{c}\text { Distribuição } \\
\text { geográica }\end{array}$ \\
\hline \multicolumn{4}{|c|}{ (EU-) DICOTILEDÔNEAS } \\
\hline Cucurbitaceae & Momordica charantia L. & Trep & Cosmop \\
\hline \multirow{7}{*}{ Euphorbiaceae } & Cnidoscolus urens (L.) Arthur & Sub & Amer \\
\hline & Euphorbia heterophylla L. & Erv & Cosmop \\
\hline & Euphorbia hirta L. & Erv & Cosmop \\
\hline & Euphorbia thymifolia L. & Erv & Cosmop \\
\hline & Jatropha gossypiifolia L. & Arb & Pantrop \\
\hline & Ricinus communis L. & Arb & Cosmop \\
\hline & Microstachys corniculata (Vahl) Griseb. & Sub & Neotr \\
\hline \multirow{6}{*}{ Fabaceae } & Crotalaria pallida Aiton & Erv & Pantrop \\
\hline & Desmodium adscendens (Sw.) DC. & Erv & Pantrop \\
\hline & Desmodium incanum (Sw.) DC. & Erv & Pantrop \\
\hline & Indigofera hirsuta $\mathrm{L}$. & Erv & Pantrop \\
\hline & Mimosa invisa Mart. ex Colla & Erv & Pantrop \\
\hline & Mimosa pudica L. & Erv & Pantrop \\
\hline \multirow{5}{*}{ Lamiaceae } & Hyptis suaveolens (L.) Poit. & Erv & Pantrop \\
\hline & Hyptis pectinata (L.) Poit. & Sub & Pantrop \\
\hline & Marsypianthes chamaedrys (Vahl) Kuntze & Sub & Neotrop \\
\hline & Ocimum basilicum $\mathrm{L}$. & Sub & Cosmop \\
\hline & Plectranthus barbatus Andrews & Sub & Gondw \\
\hline Loganiaceae & Spigelia anthelmia L. & Erv & Pantrop \\
\hline Malpighiaceae & Stigmaphyllon blanchetii C.E. Anderson & Trep & Endem \\
\hline \multirow{2}{*}{ Malvaceae } & Pavonia cancellata (L.) Cav. & Trep & Neotr \\
\hline & Waltheria indica $\mathrm{L}$. & Sub & Pantrop \\
\hline Molluginaceae & Mollugo verticillata L. & Erv & Cosmop \\
\hline Nyctaginaceae & Boerhavia diffusa $\mathrm{L}$. & Erv & Pantrop \\
\hline Onagraceae & Ludwigia octovalvis (Jacq.) P.H. Raven & Arb & Pantrop \\
\hline Passifloraceae & Turnera subulata $\mathrm{Sm}$. & Sub & Pantrop \\
\hline \multirow{2}{*}{ Piperaceae } & Peperomia pelucida (L.) Kunth & Erv & Pantrop \\
\hline & Piper aduncum L. & Arb & Pantrop \\
\hline Plantaginaceae & Scoparia dulcis L. & Erv & Pantrop \\
\hline Polygonaceae & Antigonon leptopus Hook. \& Arn. & Trep & Pantrop \\
\hline Portulacaceae & Portulaca oleracea L. & Erv & Cosmop \\
\hline \multirow{4}{*}{ Rubiaceae } & Diodella teres (Walter) Small & Erv & Cosmop \\
\hline & Richardia grandiflora (Cham. \& Schltdl.) Steud. & Erv & Neotr \\
\hline & Spermacoce capitata Ruiz \& Pav. & Sub & Pantrop \\
\hline & Spermacoce verticillata $\mathrm{L}$. & Sub & Pantrop \\
\hline \multirow{4}{*}{ Solanaceae } & Physalis angulata $\mathrm{L}$. & Sub & Cosmop \\
\hline & Solanum lycocarpum A. St.-Hil. & Arb & Endem \\
\hline & Solanum paniculatum $\mathrm{L}$. & Arb & Endem \\
\hline & Solanum stipulaceum Roem. \& Schult. & Arb & Endem \\
\hline \multirow{2}{*}{ Talinaceae } & Talinum paniculatum (Jacq.) Gaertn. & Erv & Cosmop \\
\hline & Talinum triangulare (Jacq.) Willd. & Erv & Pantrop \\
\hline Urticaceae & Cecropia pachystachya Trécul. & Árv & Endem \\
\hline \multirow{5}{*}{ Verbenaceae } & Lantana camara $\mathrm{L}$. & Sub & Cosmop \\
\hline & Lippia alba (Mill.) N.E. Br. ex Britton \& P. Wilson & Sub & Pantrop \\
\hline & Lippia gracilis Schauer & Sub & Endem \\
\hline & Priva bahiensis A. DC. & Erv & Endem \\
\hline & Stachytarpheta angustifolia (Mill.) Vahl & Erv & Gondw \\
\hline \multicolumn{4}{|c|}{ MONILOPHYTA } \\
\hline Pteridaceae & Pteris vittata $\mathrm{L}$. & Erv & Cosmop \\
\hline
\end{tabular}




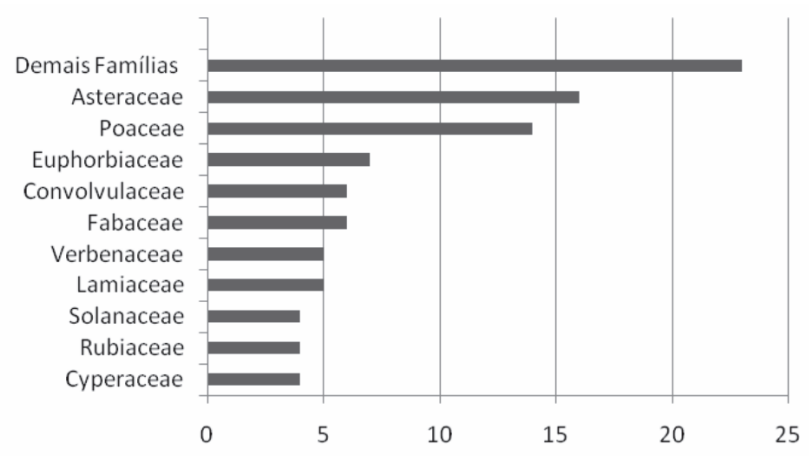

Figura 1 - Percentuais entre as famílias de plantas sinantrópicas registradas na área de estudo - João Pessoa, Paraíba.

dispersão favoráveis, como frutos alados e numerosos (van der Pijl, 1982), que favorecem a ampla colonização de grandes áreas. O estudo realizado por Silva et al. (2008), também para o Estado da Paraíba, evidenciou esse mesmo padrão, e testes com bancos de sementes feitos por Silva-Weber et al. (2012) mostraram o predomínio desses grupos.

Com relação aos tipos de hábitos dos vegetais observados (Figura 2), o herbáceo $(60,2 \%)$ foi o predominante, seguido dos hábitos subarbustivo $(19,4 \%)$, trepador $(12,2 \%)$, arbustivo $(7,1 \%)$ e arbóreo (1\%). A configuração vegetativa predominante das plantas herbáceas é bastante comum nesses ambientes perturbados, pois há ação constante de corte dessas plantas, o que impossibilitaria tempo suficiente para o desenvolvimento de vegetais de maior porte (Hill et al., 2002). Em relação à distribuição geográfica, os dados são apresentados na Figura 3.

No que se refere à análise de agrupamento (Figura 4), a partir da comparação florística das áreas analisadas, obteve-se o nível de significância de 88,3\% (coeficiente de correlação cofenética) para a interpretação dos dados.

As floras que se apresentaram separadas das demais foram as inventariadas por Marques et al. (2010) [N], que por sua vez apresentam-se vinculadas às floras estudadas por Albertino et al. (2004) [E] e Souza et al. (2003) [L], ambas localizadas na Amazônia brasileira (clima quente-úmido e elevada precipitação anual), correspondendo a culturas de feijão, guaraná e pupuaçuzeiro/pupunheira, respectivamente.
Entre as espécies compartilhadas por essas três floras, podem ser encontradas: Lantana camara, Phyllanthus niruri, Bidens pilosa, Scleria melaleuca, Vismia guianensis, Stachytarpheta cayennensis, Homolepis aturensis, Panicum laxum e Pueraria phaseoloides.

Marques et al. (2010) verificaram que as plantas sinantrópicas registradas em seu trabalho constituem um dos fatores que mais influenciaram negativamente no crescimento, no desenvolvimento e na produtividade da cultura do feijão, pois houve competição por luz (várias espécies "daninhas" arbustivas), nutrientes do solo e água, o que se reflete na redução qualiquantitativa da produção.

Para Albertino et al. (2004), o manejo das plantas "daninhas" é feito com uso de herbicidas e adubação química/orgânica. Souza et al. (2003) salientaram que os sistemas agroflorestais ou monocultivos desenvolvidos nos trópicos têm baixa produtividade por causa

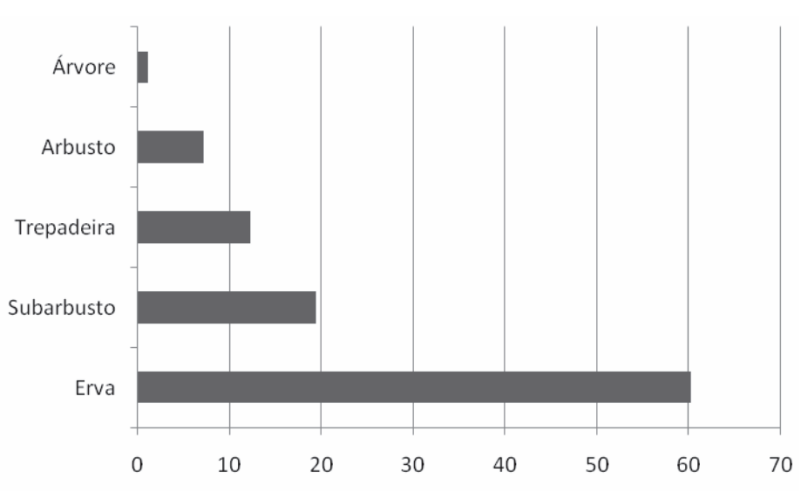

Figura 2 - Percentuais dos tipos de hábitos de plantas sinantrópicas na área de estudo - João Pessoa, Paraíba.

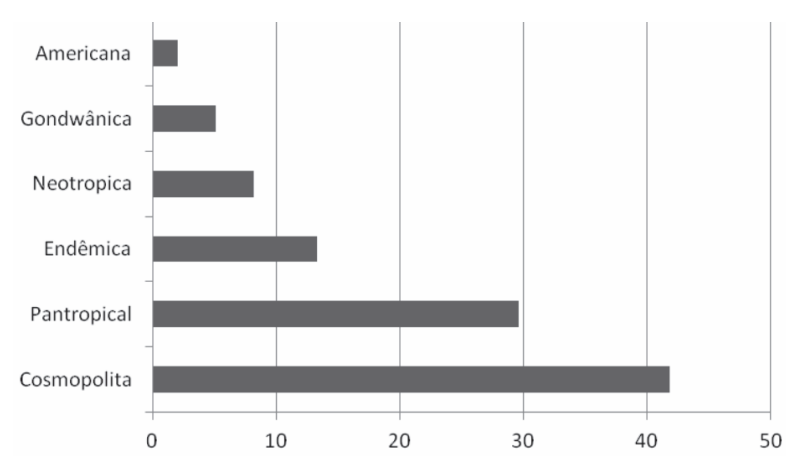

Figura 3 - Percentuais da distribuição geográfica das espécies de plantas sinantrópicas na área de estudo - João Pessoa, Paraíba. 


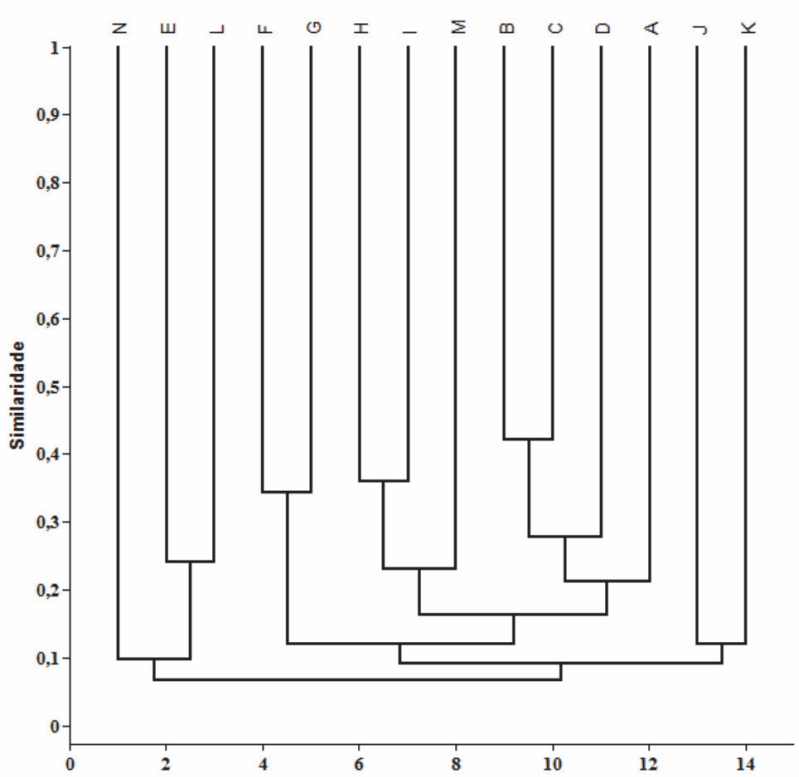

Legendas: Presente estudo [A]; Mautone et al. (1990) [B]; Gavilanes \& D'Angieri-Filho (1991) [C]; Pedrotti \& Guarim-Neto (1998) [D]; Albertino et al. (2004) [E]; Schneider \& Irgang (2005) [F]; Carneiro \& Irgang (2005) [G]; Souza \& Poletto (2007) [H]; Silva et al. (2008) [I]; Cattani (2009) [J]; Silva et al. (2006) [K]; Souza et al. (2003) [L]; Maciel et al. (2008) [M]; Marques et al. (2010) $[\mathrm{N}]$.

Figura 4 - Dendrograma de similaridade florística entre uma área periurbana no município de João Pessoa, Paraíba, e outras floras sinantrópicas, com base no índice de Sörensen.

da interferência desses vegetais. Nos monocultivos (nicho homogêneo), esses sistemas facilitam a ocupação desse grupo de plantas. Já no sistema agroflorestal (nicho heterogêneo), ocorre uma ocupação controlada, provavelmente pela competição potencial entre os táxons.

Em seguida, verificou-se o agrupamento entre as floras estudadas por Cattani (2009) [J] e Silva et al. (2006) [K]. A primeira área é uma pastagem abandonada localizada no Sudeste brasileiro, e a segunda localiza-se no Nordeste do Brasil, em uma fazenda experimental de café e grevileas no domínio semiárido.

Essa relação florística pode ser explicada, principalmente, por essas áreas estarem em regiões de clima subtropical de inverno seco (região de cerrado) e de clima tropical subúmido (região de caatinga), caracterizadas por apresentarem déficit hídrico associado à sazonalidade climática. Das espécies compartilhadas por elas, podem ser referidas: Cenchrus echinatus, Panicum maximum, Emilia sonchifolia e Senna obtusifolia.

Estudos realizados por Silva et al. (2006) e Maciel et al. (2008) detectaram que culturas com maior sombreamento por copa de árvores apresentaram uma menor riqueza de espécies sinantrópicas, quando comparadas às áreas mais abertas. Nesse contexto, Pitelli \& Pavani (2005) também confirmaram que espécies sinantrópicas toleram ambientes mais ensolarados para o seu pleno desenvolvimento e constância.

As áreas estudadas por Schneider \& Irgang (2005) [F] e Carneiro \& Irgang (2005) [G] também se apresentaram agrupadas. Essas áreas situam-se na região dos pampas gaúchos, região Sul do Brasil (clima subtropical frio e úmido, com elevada amplitude térmica), e compartilham táxons, como: Sonchus oleraceus, Chamomilla recutita, Taraxacum campylodes, Briza minor e Cynodon dactylon.

Nesse agrupamento encontram-se ambientes tipicamente perturbados pela ação antrópica, onde é generalizada a disseminação de diversas espécies invasoras, sobretudo após a alteração do equilíbrio na composição da diversidade natural dos campos sulinos do Brasil.

Ainda no dendrograma, evidencia-se a relação entre três floras totalmente inseridas em ambientes urbanos: Souza \& Poletto (2007) [H], Silva et al. (2008) [I] e Maciel et al. (2008) [M], onde as espécies mais estritamente relacionadas foram: Tridax procumbens, Bidens pilosa, Cynodon dactylon e Sporobolus indicus.

Essas áreas, apesar de estarem geograficamente distantes entre si, encontram-se inseridas em regiões planálticas: Planalto da Borborema [I] (caatinga), Planalto de Marilia $[\mathrm{H}]$ e planaltos do Oeste paulista [N] (cerrado), podendo apresentar condições ambientais relativamente semelhantes, principalmente de clima e relevo. Além disso, essas floras estão localizadas em ambientes mais urbanizados, em praças públicas, o que provavelmente as reuniu durante a análise de similaridade.

Por fim, observa-se a relação entre as floras deste estudo [A], Pedrotti \& Guarim-Neto (1998) [D], Mautone et al. (1990) [B] e Gavilanes 
\& D'Angieri-Filho (1991) [C]. O presente estudo foi caracterizado anteriormente, e as áreas [D], [B] e [C] são floras urbanas situadas no Centro-Oeste brasileiro (cerrado), em uma área serrana do Sudeste (Mata Atlântica) e no Estado de Minas Gerais, também no Sudeste do Brasil, respectivamente.

As relações florísticas observadas entre as áreas $[\mathrm{A}]$ e $[\mathrm{D}]$ foram mais distantes que as das áreas [B] e [C], porém destacam-se algumas espécies compartilhadas entre essas áreas: Alternanthera ficoidea, Amaranthus deflexus, Ageratum conyzoides, Bidens pilosa, Portulaca oleracea, Talinum paniculatum, Mimosa pudica e Euphorbia heterophylla.

A flora sinantrópica do presente estudo [A] encontra-se inserida em uma localidade tipicamente tropical com influência do oceano Atlântico e está relacionada, entre as áreas selecionadas para a análise, a uma área do Centro-Oeste brasileiro [D], compartilhando as espécies: Tridax procumbens, Euphorbia hirta, Boerhavia diffusa, Lippia alba, Porophyllum ruderale, Commelina benghalensis, Dactyloctenium aegyptium, Cenchrus echinatus e Digitaria ciliaris.

Entre as floras [B] e [C], que se apresentaram como as mais similares neste estudo, provavelmente pela proximidade geográfica entre elas, destacaram-se as espécies: Cosmos caudatus, Ageratum conyzoides, Momordica charantia, Portulaca oleracea, Talinum paniculatum, Euphorbia heterophylla, Bidens pilosa, Amaranthus deflexus, Cyperus rotumdus, Sonchus oleraceus e Eleusine indica.

Com base nos resultados obtidos e na hipótese levantada, apesar de as espécies no geral apresentarem ampla distribuição geográfica, houve agrupamentos particulares associados às condições regionais (domínio amazônico, pampeano, cerrado-caatinga, agrupamento de floras assentadas em áreas de "altitude" e tipicamente urbanizadas).

Apesar da distância geográfica entre as áreas antropizadas, nos agrupamentos $[\mathrm{I}]-[\mathrm{H}]-$ $[\mathrm{N}]$ e $[\mathrm{A}]-[\mathrm{D}]-[\mathrm{B}]-[\mathrm{C}]$, áreas que estavam mais separadas geograficamente entre si exibiram maior conexão florística independentemente da região biogeográfica, pelo fato de compartilharem várias espécies do tipo cosmopolita e por apresentarem um micro-habitat mais semelhante (urbanizado).

Segundo Hill et al. (2002), as zonas urbanas promovem várias formas de alterações no ambiente natural, como a homogeneização do habitat. Esses fatores, adicionados às características de espécies vegetais não nativas e/ou cosmopolitas, acabam facilitando aglomerações vegetacionais mais similares entre si. Schneider \& Irgang (2005) enfatizam que, quanto mais perturbado o ambiente espacial, historicamente maior será a similaridade florística entre áreas nessa equivalência.

Desse modo, apesar de as espécies sinantrópicas serem consideradas agrupamentos vegetacionais de espécies generalistas e cosmopolitas, há formação de grupos específicos em zonas tipicamente rurais e urbanizadas, evidenciando que, embora vários táxons sejam compartilhados entre essas áreas, especialmente nos niveis de família e gênero, as formações de nichos específicos preenchidos por espécies diferentes demandam condições ambientais e recursos também diferentes.

\section{AGRADECIMENTOS}

J.I.M. Melo agradece ao Conselho Nacional de Desenvolvimento Científico e Tecnológico (CNPq) a Bolsa de Produtividade em Pesquisa (Proc. $n^{0}$. 302751/2012-2).

\section{LITERATURA CITADA}

AESA. Online [Internet]. Agência Executiva de Gestão das Águas do Estado da Paraíba. Disponível em: <http:// site2.aesa.pb.gov.br/aesa/

medicaoPluviometrica.do?metodo=chuvasDiariasMapa $>$. Acessado em: 14 dez. 2013.

ALBERTINO, S. M. F. et al. Composição florística das plantas daninhas na cultura de guaraná (Paullinia cupana), no Estado do Amazonas. Planta Daninha, v. 22, n. 3, p. 351-358, 2004.

ALMEIDA, J. D.; FREITAS, H. A flora exótica e invasora de Portugal. Portugaliae Acta Biol., v. 16, n. 1, p. 159-176, 2000.

APG III. An update of the Angiosperm Phylogeny Group classification for orders and families of flowering plants: APG III. Bot. J. Linnean Soc., v. 161, n. 2, p. 105-121, 2009. 
ARANHA, C. et al. Sistemática de plantas invasoras. Campinas: Instituto Campineiro de Ensino Agrícola, 1988. $174 \mathrm{p}$.

BARROSO, G. M. et al. Sistemática de angiospermas do Brasil. São Paulo: Universidade de São Paulo, 1978. v. 1. $255 \mathrm{p}$.

BARROSO, G. M. et al. Sistemática de angiospermas do Brasil. Viçosa, MG: Universidade Federal de Viçosa, 1984. v. 2.377 p.

BARROSO, G. M. et al. Sistemática de angiospermas do Brasil. Viçosa, MG: Universidade Federal de Viçosa, 1986. v. $3.326 \mathrm{p}$.

BRIDSON, D.; FORMAN, L. The herbarium handbook. Kew: The Royal Botanic Garden, 2004. 334 p.

CARNEIRO, A. M.; IRGANG, B. E. Origem e distribuição geográfica das espécies ruderais da Vila de Santo Amaro, General Câmara, Rio Grande do Sul. Iheringia, Série Bot., v. 60, n. 2 , p. $175-188,2005$.

CATTANI, S. M. M. Levantamento de espécies ruderais em uma área de pastagem abandonada na Represa de

Itupararanga, Votorantim-SP. R. Eletr. Biol., v. 2, n. 4, p. 38-55, 2009.

COLAUTTI, R. I.; MACISAAC, H. J. A neutral terminology to define 'invasive' species. Diver. Distrib., v. 10, n. 1, p. 135-141, 2004.

FORZZA, R. F. et al. Online [Internet]. Lista de espécies da flora do Brasil. Disponível em: <http://reflora.jbrj.gov.br/ jabot/listaBrasil/ConsultaPublicaUC/ConsultaPublicaUC.do>. Acesso em: 14 set. 2013.

GAVILANES, M. G.; D’ANGIERI-FILHO, C. N. Flórula ruderal da cidade de Lavras, Minas Gerais. Acta Bot. Bras., v. 5, n. 2, p. 77-88, 1991.

GLOBAL BIODIVERSITY INFORMATION FACILITY. Online [Internet]. Free and Open Access to Biodiversity Data. Disponível em: <23http:// www.gbif.org/>. Acesso em: $12 \mathrm{dez}$. 2013.

GLOBAL INVASIVE SPECIES DATABASE. Online [Internet]. Standard search. Disponível em: $<$ http:// www.issg.org/database/1>. Acesso em: 3 set. 2013.

GONÇALVES, E. G.; LORENZI, H. Morfologia vegetal. Nova Odessa: Instituto Plantarum de Estudos da Flora, 2012. $447 \mathrm{p}$.

GOTELli, N. J.; ELLISON, A. M. Princípios de estatística em ecologia. Porto Alegre: Artmed, 2011. 527 p.
HASSEMER, G.; TREVISAN R. Levantamento florístico de plantas vasculares espontâneas em ambientes antrópicos no campus da Universidade Federal de Santa Catarina.

R. Biotemas, v. 25, n. 3, p. 75-96, 2012.

HILL, M. O. et al. Hemeroby, urbanity and ruderality: bioindicators of disturbance and human impact. J. Applied Ecol., v. 39, n. 1, p. 708-720, 2002.

JAROLÍMEK, I.; ZALIBEROVÁ, M. Ruderal plant communities of north-eastern Slovakia II. Thaiszia, v. 5, n. 1, p. 61-79, 1995.

JSTOR PLANT SCIENCE. Online [Internet]. JSTOR Plant Science. Disponível em: <http://plants.jstor.org>. Acesso em: 3 set. 2013.

KENT, M.; COKER, P. Vegetation description and analysis: A practical approach. Chichester: John Wiley, 1995. $428 \mathrm{p}$.

KISSMANN, K. G.; GROTH, D. Plantas infestantes e nocivas. 2.ed. São Paulo: BASF, 1997. Tomo I. 825 p.

KISSMANN, K. G.; GROTH, D. Plantas infestantes e nocivas. 2.ed. São Paulo: BASF, 1999. Tomo II. 978 p.

KISSMANN, K. G.; GROTH, D. Plantas infestantes e nocivas. 2.ed. São Paulo: BASF, 2000. Tomo III. 726 p.

LORENZI, H. Plantas daninhas do Brasil. 4.ed. Nova Odessa: Instituto Plantarum de Estudos da Flora, 2008. 672 p.

MOBOT. Online [Internet]. Missouri Botanical Garden (W³ Tropicos). Disponível em: <http://www.mobot.org/w3 search/vast.html $>$. Acessado em: 3 set. 2013.

MACIEL, C. D. G. et al. Composição florística da comunidade infestante em gramados de Paspalum notatum no município de Assis, São Paulo. Planta Daninha, v. 26, n. 1, p. 57-64, 2008.

MARQUES, L. J. P. et al. Composição florística de plantas daninhas na cultura do feijão-caupi no sistema de capoeira triturada. Planta Daninha, v. 28, p. 953-961, 2010. (Número Especial)

MAUTONE, L. et al. Daninhas ocorrentes na zona serrana do estado do Rio de Janeiro - município de Petrópolis. Acta Bot. Brasilica, v. 4, n. 2, p. 123-135, 1990.

MENNAN, H.; ISIK, D. Invasive weed species in onion production systems during the last 25 years in Amasya, Turkey. Pakistan J. Bot., v. 35, n. 2, p. 155-160, 2003.

PEDROTTI, D. E.; GUARIM-NETO, G. Flora ruderal da cidade de Cuiabá, Mato Grosso, Brasil. Acta Bot. Brasilica, v. 12, n. 2, p. 135-143, 1998.

Planta Daninha, Viçosa-MG, v. 33, n. 1, p. 57-66, 2015 
PIŠEK, P. et al. Alien plants in checklists and floras: towards better communication between taxonomists and ecologists. Taxon, v. 53, n. 1, p. 131-143, 2004.

PITELLI, R. A.; PAVANI, M. C. M. D. Feralidade vegetal e transgeníese, evolução adaptativa das plantas invasoras. Biotecnol. Ci. Desenvol., v. 34, n. 1, p. 100-104, 2005.

RAJPUT, M. T. et al. Check list of the weeds found in cotton crops, cultivated in Taluka Ubauro, district Sukkur, Pakistan. Pakistan J. Bot., v. 40, n. 1, p. 65-70, 2008.

SCHNEIDER, A. A.; IRGANG, B. E. Florística e fitossociologia de vegetação viária no município de Não-MeToque, Rio Grande do Sul, Brasil. Iheringia, Série Bot., v. 60, n. 1, p. 49-62, 2005.

SHAN-HUAH, W. U. et al. Patterns of plant invasions in the preserves and recreation areas of Shei-Pa National Park in Taiwan. Bot. Studies, v. 50, n. 1, p. 217-227, 2009.

SILVA, S. O. et al. Diversidade e frequência de plantas daninhas em associações entre xafeeiros e grevíleas. Coffee Sci., v. 1, n. 2, p. 126-134, 2006.

SILVA, A. M. A. et al. Levantamento florístico das plantas daninhas em um parque público de Campina Grande, Paraíba, Brasil. R. Biot., v. 21, n. 4, p. 7-14, 2008.
SILVA, M. A. et al. Levantamento das plantas espontâneas e suas potencialidades fitoterapêuticas: um estudo do complexo Aluízio Campos - Campina Grande - PB. R. Bras. Inf.

Cient., v. 1, n. 1, p. 52-66, 2010.

SILVA-WEBER, A. J. C. et al. Composição florística e distribuição sazonal do banco de sementes em Floresta Ombrófila Mista Aluvial, Araucária, PR. Pesq. Flor. Bras., v. 32, n. 70, p. 77-91, 2012.

SOUZA, A. A.; POLETTO, R. S. Levantamento de espécies invasoras nas praças de Graça - SP - Magnoliopsida e Liliopsida. R. Cien. Eletr. Eng. Flor., v. 1, n. 9, p. 1-14, 2007.

SOUZA, R. P.; VALIO, I. F. M. Seed size, seed germination and seedling survival of Brazilian tropical trees species differing successional status. Biotropica, v. 33, n. 3 , p. 447-457, 2001.

SOUZA, L. S. A. et al. Composição florística de plantas daninhas em agrossistemas de cupuaçuzeiro (Theobroma grandiflorum) e pupunheira (Bactris gasipaes).

Planta Daninha, v. 21, n. 2, p. 249-255, 2003.

THE PLANT LIST. Online [Internet]. The plant list. Disponível em: <http://www.theplantlist.org/>. Acessado em: 12 out. 2013.

Van der PIJL, L. Principles of dispersal in higher plants. 3.ed. New York: Springer-Verlag, 1982. 215 p. 